\title{
A Reference Architecture for Sharing CDA Documents in
}

\section{Colombia}

\author{
Edgar De la Cruz ${ }^{1}$, Diego M. Lopez ${ }^{1,2}$ Bernd Blobel $^{2}$ \\ ${ }^{1}$ Telematics Department, University of Cauca, Colombia \\ ${ }^{2}$ eHealth Competence Center Regensburg, Germany
}

\begin{abstract}
Background: The Colombian health system is complex. There are more than 3200 healthcare service providers aiming at offering quality of care to more than 48 million citizens. Unfortunately, health services are not integrated, a citizen has a fraction of its health record in the different health services provider organizations without a chance for integration. Objective: The objective of this paper is to propose a reference architecture for an integrated Electronic Health Record System in Colombia based on the ISO/HL7 CDA standard. Methods: A formal system architecture is defined based on the Health Information Systems Development Framework (HIS-DF). Results: The architecture is described as platform independent architecture expressed by three different models: The enterprise model describing business process and policies, the information model describing dynamic and static information flows, and the computational model, describing the systems components and interrelations, based on software engineering architectural styles and patterns. Conclusions: The proposed architecture has been compared with traditional architectural models for Integrated Electronic Health Record Systems. The architecture was evaluated through a case study in dental care were the availability, chronology, completeness and timeliness of the EHR stored in the system were considered high Also perceived quality was high as evaluated by the users.
\end{abstract}

\section{Keywords}

Health information systems, system integration, Electronic Health Records, semantic interoperability, Clinical Document Architecture, HL7, CDA, EHR.

\section{Correspondence to:}

Prof. Dr. Diego M. Lopez

Telematics Department, University of Cauca, Colombia

Calle 5 No 4-70

E-mail: dmlopez@unicauca.edu.co

EJBI 2012; 8(3):11-17

\section{Introduction}

In the Colombian health system, there are more than 80 insurance companies (Health Promotion Entities EPS) responsible for the administration of healthcare services through their contractual relationships with more than 3200 different healthcare service providers (Healthcare Provision Institutions, IPS); the latter includes clinics, hospitals, laboratories, imaging services, etc. [1]. In this context, healthcare services provision is not supported by a single IPS, each citizen has a fraction of its health record in the different IPS he has visited through his life. If health records are not integrated, continuity of care is hardly supported.

According to Colombian law, a health record is defined as a private document, mandatory, and subject to clinical protection, which chronologically registers health conditions, medical procedures and other procedures performed by healthcare teams to a patient [2]. One of the basic features of medical records according to this legislation is its availability, which means access to medical information at the time it is need. However, when a patient has to move from one healthcare institution to another, the availability of information is limited. This happens because IPS typically have no direct access to the fraction of medical records stored in other IPS. Therefore, unless 


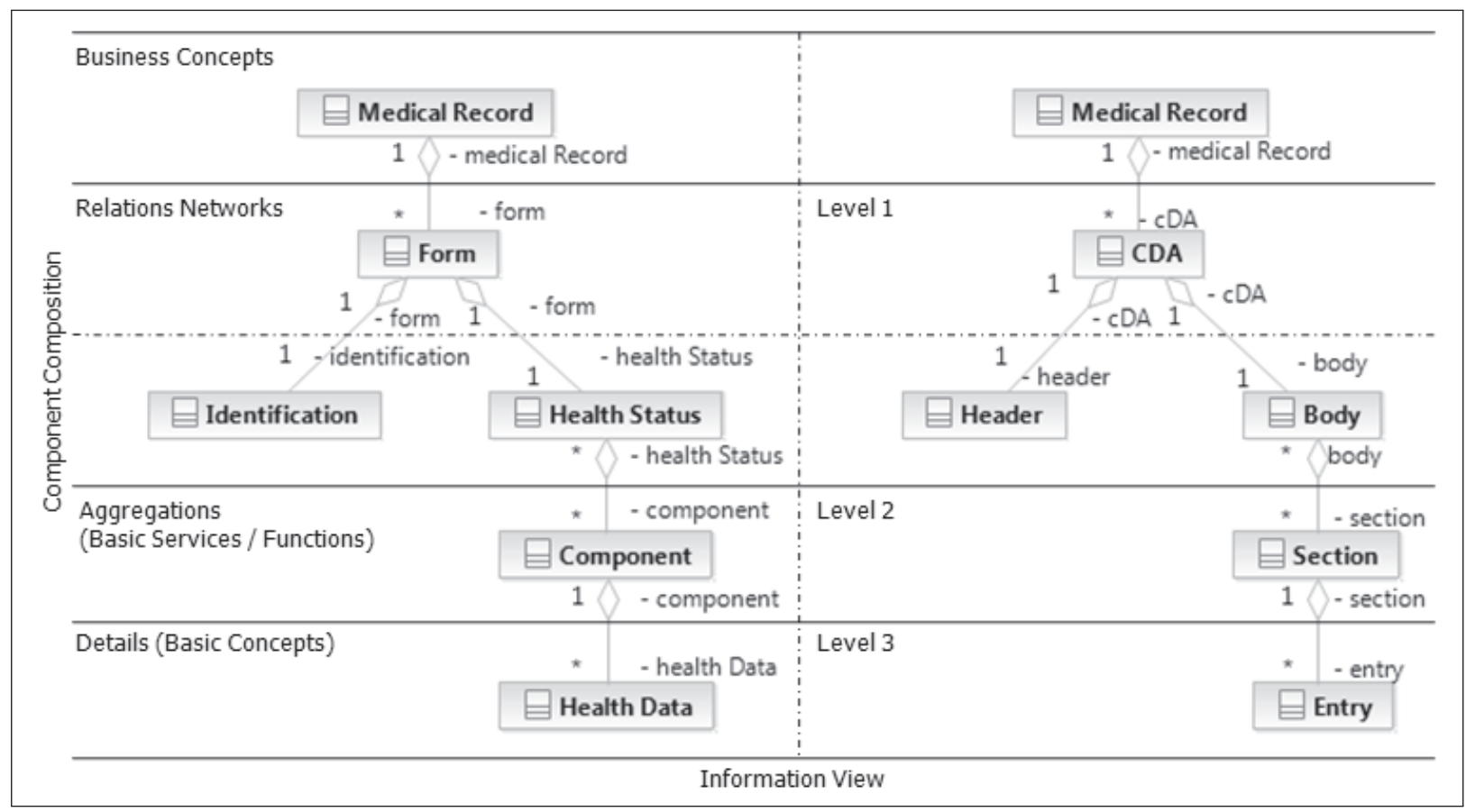

Figure 1: Comparing the composition of a generic Medical Record as well as CDA documents using the GCM

the patient (concerned about his own health) takes responsibility for sharing paper copies of his own record, the caregiver has to rely on the information patient is able to communicate. Another feature that is compromised when health records are not integrated is its chronology, meaning that records must have a correct chronological register of the care provided. Considering the complexity of the Colombian health system, to achieve this availability and chronology is a very complex task, since information is distributed among the different IPS. Therefore, it is not easy at any given time to integrate the different sources of information.

A feasible solution to the aforementioned challenges is the availability of Electronic Health Record Systems (EHR-S), able to electronically manage citizens' health information. The next step is to facilitate interoperability between EHR-S through the provision of a nation-wide Integrated Electronic Health Record System (I-EHRS). Especially big healthcare providers have already implemented EHRS in Colombia. However, two of the main challenges to be overcome in the country in order to accomplish that national I-EHRS are a) to provide standardized models for clinical information exchange, as each system stores data internally in a different format, and b) provide the ICT infrastructure to enable information sharing among different institutions [3]. Some international interoperability standards specifically for Electronic Health Records interoperability are in place, such as de ISO/HL7 Clinical Document Architecture (CDA), the ISO13606, openEHR Archetypes, and more recently the Detail Clinical Models (DCM) approach (ISO 13972). Unfortunately, many of these standards are not widely used because they are normally unknown, complex, overlapping, incomplete and lack of specialized tools for their implementation [4]. Keeping in mind that selecting a standard language for enabling healthcare interoperability is not sufficient to ensure semantic interoperability unless this standard is agreed to be used by all actors involved in citizens' care, it is suggested that at the first stage of an interoperability project a single EHR standard be adopted (at least) at national level [5]. In Colombia, the only and most mature work performed in the area of standardization of health information is being developed by HL7 Colombia, which has recommended using CDA Release 2 as standard for EHR [6].

The objective of this paper is to propose a reference architecture for an integrated EHR system in Colombia based on the CDA standard. As demonstrated in other countries, a project at such scale succeeds only if the formal design of the system's architecture is first provided $[7]$.

\section{Materials and Methods}

After analyzing main computational approaches for integrated EHR architectures, three types of architectural models were identified: a) centralized model, where the clinical documents are stored in a centralized repository, b) distributed model, which is a fully distributed architecture, where each healthcare service provider has its own clinical documents repository and c) hybrid model, which uses distributed repositories supported by a centralized indexing metadata service. The following disadvantages of the different approaches were found:

- Centralized indexing and centralized repositories are 


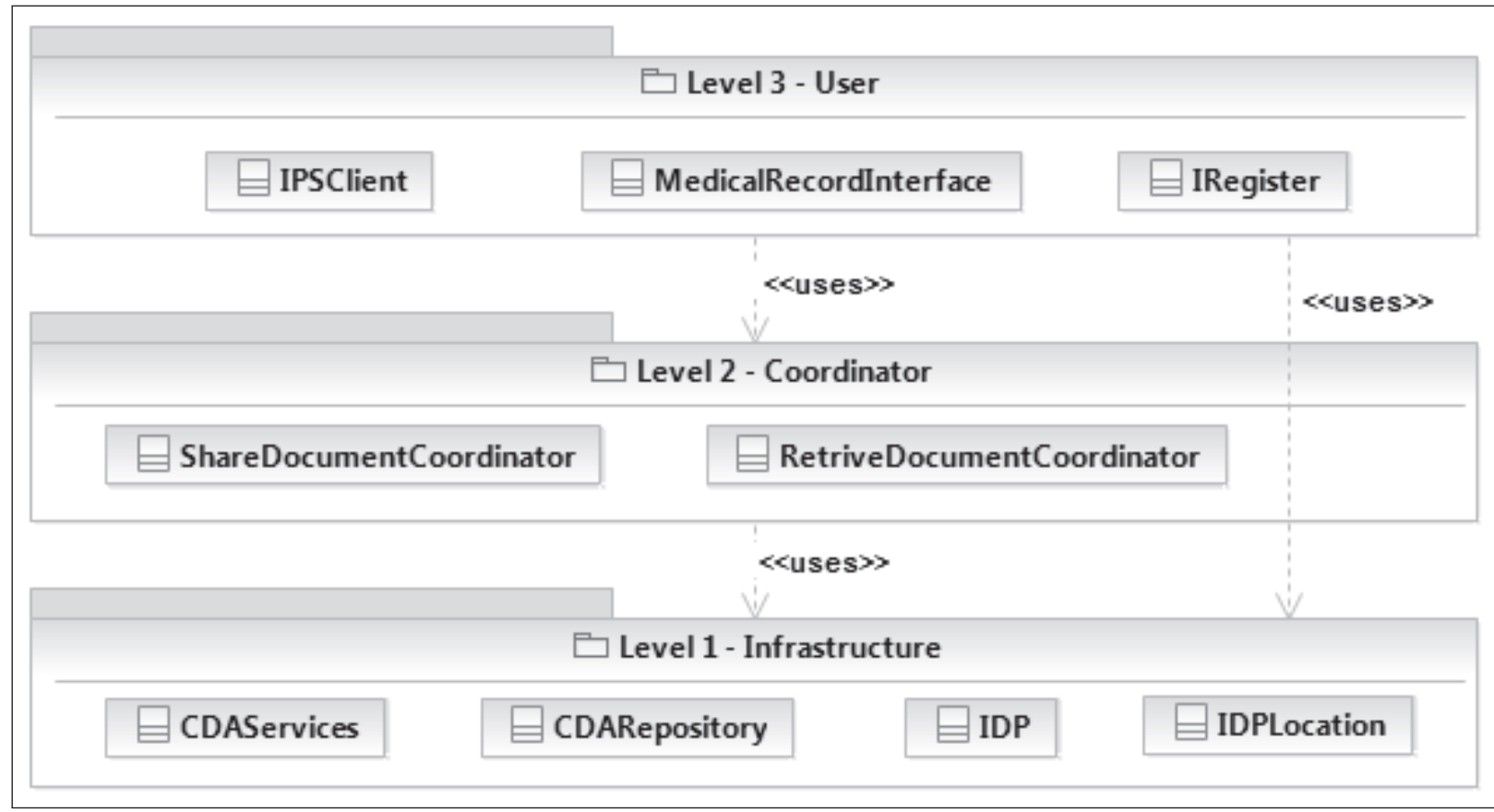

Figure 2: Tiered architecture for Integrated Medical Record System

allocated in a central point. Therefore, any failure affects the availability and reliability of the entire system.

- In a distributed architectural model, as each healthcare service provider creates and stores clinical documents in its own repository, it would be necessary that after the new document is created and locally stored, the other service providers (nodes) be notified about the existence of the new clinical document.

- Most of the approaches are not based on EHR standards.

- A detailed description of infrastructure required for sharing EHR is not provided, only [8, 9] considers the infrastructure, but this approach does not support any EHR standard.

The proposed architecture for the Colombian I-EHRS is designed as a platform-independent architecture based on the Health Information System Development Framework (HIS-DF) methodology [10]. The methodology describes the architecture as a set of three interrelated views on that system: the business, the information and the computational models. This goes beyond the computational models above presented.

\section{Results}

The business viewpoint defines the objective, scope and policies of the organization, especially considering existing national legislation related to health records, e.g., Green Book on Medical Records [11], Agreement 07 of
1994 [12], Resolution 2546 of 1998 [13], Resolution No. 1995 of 1999 [2], and Act 1438 of 2011 [14]. The main policy is Resolution No. 1995 of 1999 which establishes some requirements for paper based health records, naming the health care service providers as custodians, and the patient as the owner of the information. Basic confidentiality policies are also declared. It is also mentioned that information can be electronically managed keeping security and confidentiality. The recent Act 1438 of 2011 establishes that EHR has to be implemented by January 2014, however no details or governmental support has been defined for that.

In the information viewpoint, models describing the information to be stored and processed in the integrated EHR system are presented. It includes documentation about a patient's health status as well as metadata for the identification of clinical documents, indexing information and user data as basic information. In this model, the CDA standard provides guidance for defining structure and semantics of clinical documents and metadata. presents the structure of medical records as defined in the Colombian health system, compared with the hierarchy of documents established by CDA according to the composition/decomposition levels defined by the Generic Component Model (GCM) [7, 10] (see Figure 1). The Information viewpoint was completed by the development of a CDA Release 2 implementation guide for general dentistry.

The Computational viewpoint defines the components of the integrated EHR system, which interact through interfaces. The components are structured following a tiered architectural style [15], so that each component is at a level where it is possible to obtain services from a lower level, but not from a higher level. Figure 2 shows the 
tiered architecture for integrated medical record system using the tiered architecture style. Notice that the levels depicted in Figure 2 do not correspond to the ones in Figure 1, as the first describes relations between independent service systems while the latter presents a composition/decomposition process of one system (aggregation/specialization of the system's components).

To manage the interaction between the different components of the architecture, the following communication patterns were applied:

- Synchronous message communication - response: a typical communication pattern of the client / server model, where the client expects the server's response before continuing its tasks [16]. This pattern is used to communicate between clients at the User Level (level 3) and services at the Coordinator Level (Level 2).

- Broker Handling: this pattern is typically used to provide location transparency to users requesting services in a service-oriented architecture (SOA) [16]. In the proposed architecture, this approach is used to allow services at the Coordinator Level (Level 2) to locate services of the Infrastructure Level (Level 3), in order to respond to users' request (Level 1).

The functionality of the different services is summarized as follows:

- IPSClient: this component hides the details on how to access a legacy database of a specific IPS. It is also responsible for continually checking whether a new clinical document has been created, in order to create an interface for quering the legacy system using SQL, XML, etc. to retrieve the corresponding data and to compile them into a message that is sent to the ShareDocumentCoordinator.

- ShareDocumentCoordinator: this component mediates between the Client IPS and other components of the architecture. Its function is to coordinate the workflow in the process of sharing clinical documents as follows: i) IPSClient sends a message with the necessary parameters to create the CDA document, ii) it verifies that the user exists in the system and if so, it assigns an identifier (Indexing of Documents by Patient, IDP) after invoking the IDPLocation component, iii) it verifies that the document to be generated has not been stored previously in the repository, iv) it generates the CDA through the CDAServices component, v) it stores the CDA document into the CDARepository component associated with the IPS that generated it, vi) it requests the overall user identification to the IDPLocation component, vii) it reports the existence of a new document to the IDP component.

- CDAServices: it receives from the ShareDocumentCoordinator component the parameters necessary to create the CDA document. When being ready, it creates, validates and returns a document in CDA Release 2 format.

- CDARepository: It is used to store clinical documents generated by the CDAServices component. It also checks that the CDA document has not been previously stored.

- Indexing of Documents by Patient (IDP): this component provides information on how to locate any document of any specific patient. This component allows retrieving all the metadata associated with the patient's health record through a universal patient identification.

- IDPLocation: this component stores the basic data for a patient together with the IDP assigned at the time of registration, information about physicians authorized to access the EHR. When invoked, it returns a URL. Despite of multiple local identifiers assigned to a patient, a global identifier is maintained.

- Registration Interface - IRegister: it allows creating users' accounts. When the user is registered, an IDP is assigned to the patient and is reported to the IDPLocation component.

- RetrieveDocumentCoordinator: its function is to coordinate the sequence of components used to retrieve a clinical document. The steps for this process are: i) receive the user ID from the MedicalRecordInterface component when the user intends to consult a medical record; ii) requests from the IDPLocation component the IDP of any given patient, iii) obtain the global identification of the patient using the IDPLocation component, iv) obtain the metadata of the patient's clinical document, and v) return the metadata to the MedicalRecordInterface component.

- Privacy, Access and Security Services - PASS: it is a service for managing privacy and access control based on HL7-SOA specifications.

- Medical Record Interface: it presents the user interface used by healthcare professionals and citizens to interact with the system. Using the patient's identification, it chronologically displays the different medical records. It is responsible for retrieving the clinical document from the CDA Repository component using the metadata information.

For implementing the proposed, platform-independent architecture, different technologies can be used for each of the proposed components. Following technologies have been used for the practical implementation of the proposed solution:

- Java was used as Programing Language. It was selected as it is an object oriented language providing platform independent services' implementation. 


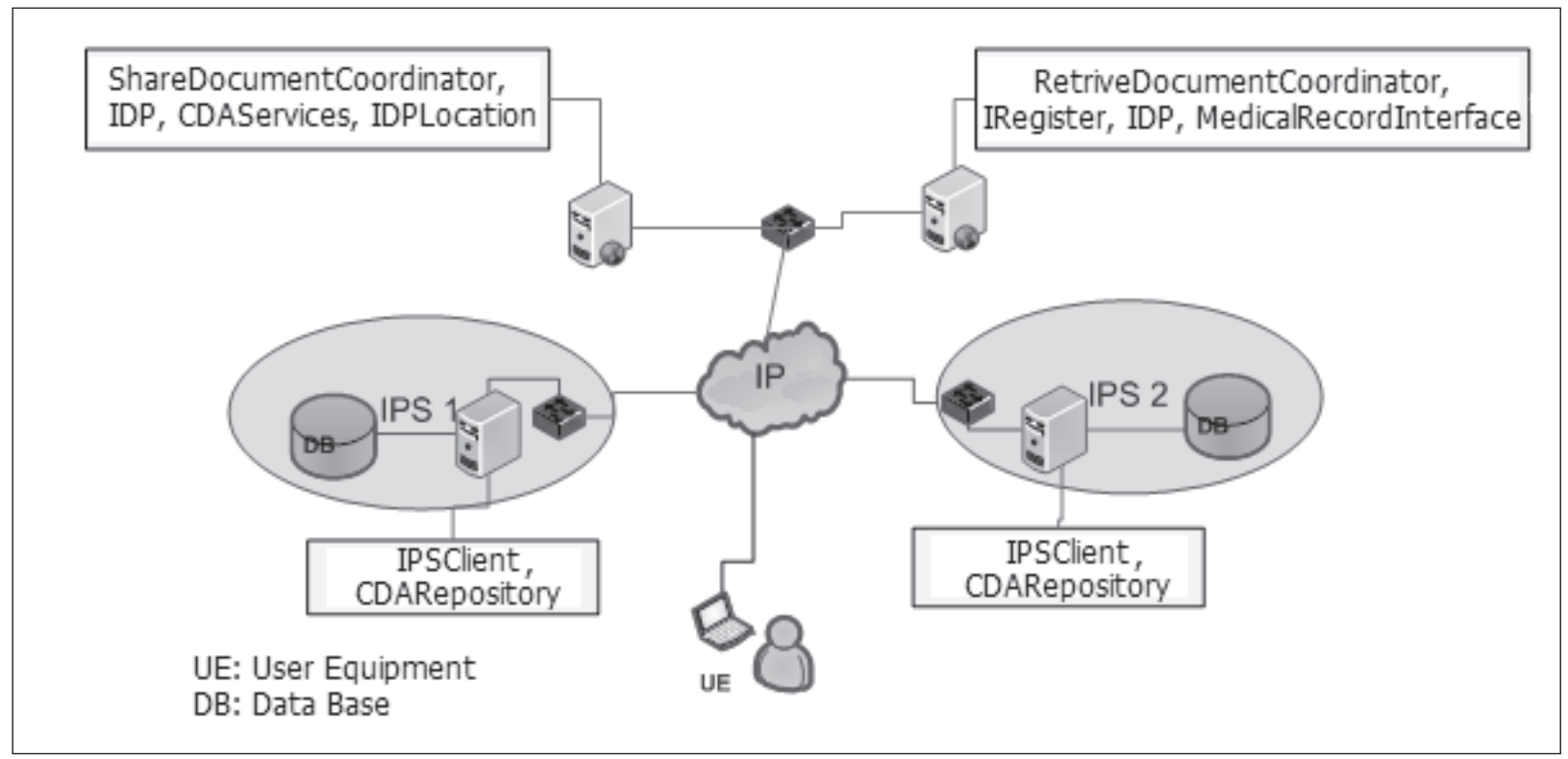

Figure 3: System Deployement Model

- The Model-Driven Health Tools (MDHT) CDA project was used to create and validate the CDA documents. The project is part of the Eclipse Open Health Tools project, and is able to create a CDA from an existing CDA implentation guide.

- Apache Axis2 provides a library to implement both the client and server Web services. It provides support for standards such as SOAP, REST, WSDL, etc.[17]. Apache Axis2 version 1.5.6 was used for implementing the components in the Coordinator Level (Level 2).

- MySQL is a relational database which supports multi-threaded, multi-user server and SQL. MySQL is offered under the GNU GPL licensing model, but it is possible to contract a commercial license with Oracle [18]. MySQL version 5.1 was used in the implementation of the following components: IDPLocation component to store the user log data shared EHR system; IDP component to store documents indexed by clinical and patient identification; CDARepository component to store only the unique identifier of stored documents, and DocumentSharingCoordinator component to store the repository's address and CDA associated with each of the IPS to be used by this component.

- Apache Tomcat is an open source implementation of Java Servlet and Java Server Pages [19]. Tomcat 5.5 was used to deploy the different components of the architecture. It was also used to store CDA documents, so they can be accessed via the Internet.

- Apache is an open source project used to deploy Web Servers. Apache 2.2.16 was used as a proxy to forward requests to port 80 for the Tomcat Server service. It allows Web services to be accessed via port 80 preventing problems with blocked ports.

- ICEfaces is an open source project, which provides JSF (Java Server Faces) and Ajax components, simplifying the development of user interfaces [20].

- XSLT is a W3C standard used to transform an XML document into another format [21]. In the architecture, it takes as input the XML of the CDA document and uses a XSL Style Sheet Transformation to create a web page that displays document information to clinical health specialist. The XSLT engine used is the browser Firefox 12 .

Figure 3 shows the deployment model of the reference implementation of the architecture.

\section{Discussion}

The method proposed by Kitchenham and Pickard [22] for the evaluation of software engineering artifacts through case studies was used to evaluate the reference implementation. The use case evaluation comprises the following activities:

- Interoperability agreement. A formal agreement was established between the institutions taking part in the study. The institutions are two IPS in the domain of dental care located in the city of Popayan, Colombia: Smisalud and Unidad de Salud. The latter is the medical service for faculty and employees at University of Cauca.

- Agreement on the clinical documents to be integrated. General Dentistry and Emergency documents were chosen for communication. 


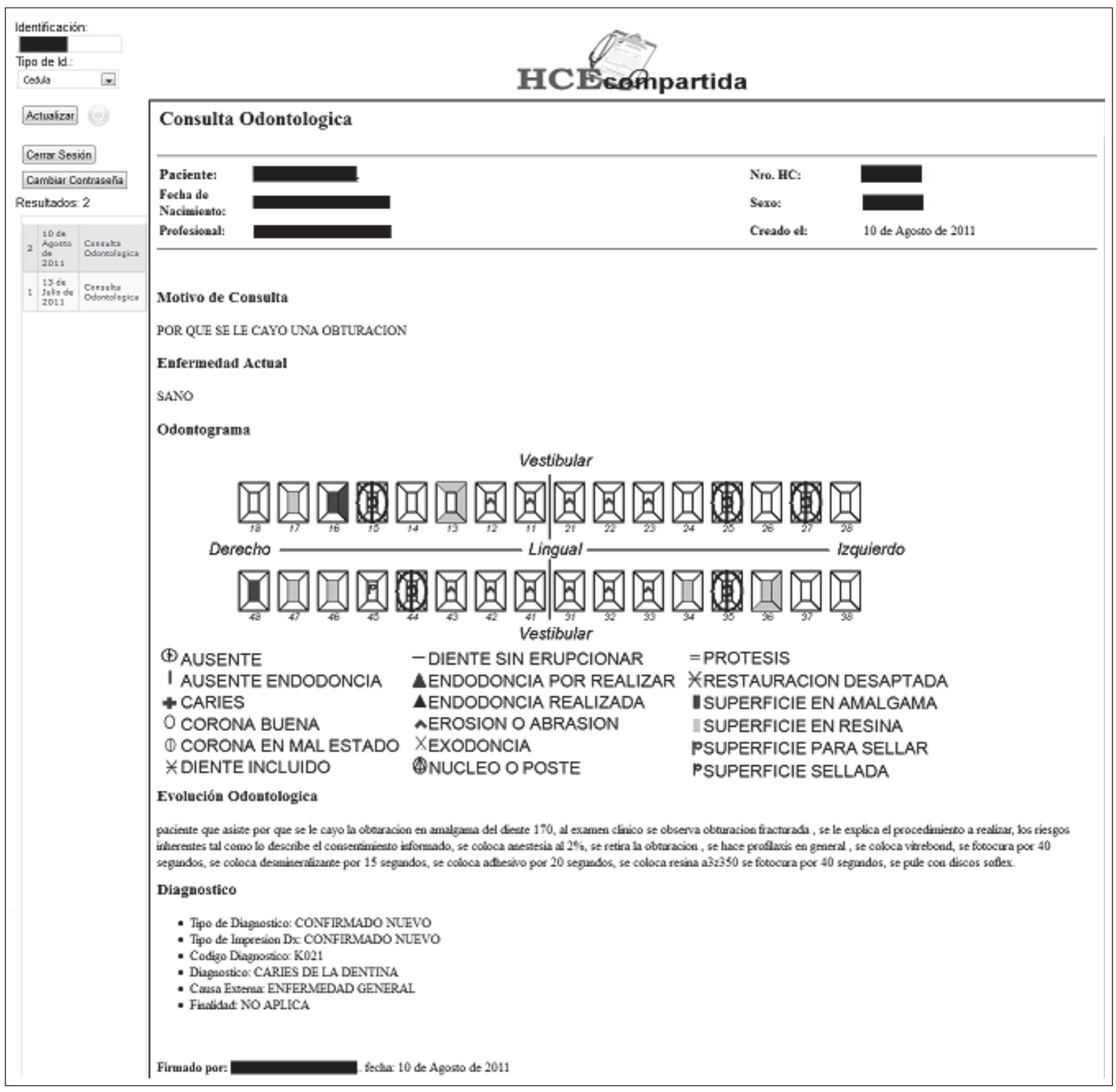

Figure 4: Example of a clinical document displayed by the MedicalRecordInterface component

- Define the evaluation tools and instruments to be used in the pilot evaluation.

- Connect the EHR Systems of the two IPS

- Obtain an informed consent of the patients who agree to participate in the study.

- Upload the EHR of the patients participating in the study into the system. The clinical documents are uploaded to the Integrated EHR system.

- Analyze and report the results. This includes:

- Assess the availability, chronology, completeness, timeliness of the EHR of patients who agreed to participate in the case study.

- Evaluate the perceived quality of the system by users.

\section{Example of a clinical document displayed by the MedicalRecordInterface component}

Figure 4 shows an example of a clinical document displayed by the MedicalRecordInterface component.

Thirty patients participated in the study, but only sixteen responded to a questionnaire asking about the perceived utility of the system. Overall; the perceived utility was assessed as very high $(81,25 \%)$ and high $(18,75 \%)$. A Dental hygienists at unidad de Salud was asked about the availability, sequentially, completeness, timeliness of the electronic dental records stored in the system for the thirty patients taking part in the study. The overall avail- 
ability sequence, completeness, timeliness was evaluated as $100 \%$. The Dental Hygienist was also asked about the usability of the system, responding that it is very easy to use; he was asked about the perceived utility of the system, answering that the system's utility is very high, especially important for accessing to the information independent of place and time; and asked about the impact of the system in his daily work, claiming that it's very important for supporting diagnosis, check the sequentially of treatments, obtaining an accurate register of the patient's dental record, and also improving the quality of the records.

\section{Conclusions}

The proposed architecture has the following advantages compared with other architectures for integrated health records:

- It provides a distributed approach indexing metadata of clinical documents per patient. By that way, it increases system reliability because of the robustness of the system in cases a component fails.

- The architecture is presented as platformindependent, thereby providing implementation flexibility.

- It is scalable, allowing to share a common infrastructure with different EHR systems.

- It supports the specific requirements for electronic medical records in Colombia.

The architecture was evaluated through a case study were the availability, chronology, completeness and timeliness of the EHR stored in the system were considered high. Also the perceived quality was high as evaluated by the users.

\section{Acknowledgements}

The work was partially funded by DAAD, Colciencias and University of Cauca (RC No 567-2011), CYTEDCNPq (SALUS), Colciencias Program jóvenes investigadores (call 510/10) and QUIPU Program under US National Institutes of Health Fogarty grant D43TW008438, and University of Cauca in Colombia.

\section{References}

[1] Gorbaneff Y, Torres S and Contreras N. Anatomía de la cadena de prestación de salud en Colombia en el régimen contributivo. Innovar. 2004; 14:, 23.

[2] Ministerio de Salud. Resolución Número 1995 de 1999. 1999.

[3] Wu C and Hadzic M. Creating Interoperability Within Healthcare Industry, 2008.
[4] López DM and Blobel B. Enhanced semantic interoperability by profiling health informatics standards. Methods Inf Med. 2009; 48(2): 170-177.

[5] Benson T. Principles of health interoperability HL7 and SNOMED. Heidelberg: Springer Verlag; 2010.

[6] HL7 Colombia. Fundación HL7 Colombia, 2009. [Online]. Available: http://www.hl7.org.co/nuevo/. [Accessed: 21-ene2011].

[7] Blobel B and Pharow P. Analysis and evaluation of EHR approaches. Meth Inf Med. 2009: 48(2): 170-177.

[8] Katehakis DG, Sfakianakis SG, Kavlentakis G, Anthoulakis DN and Tsiknakis M. Delivering a lifelong integrated electronic health record based on a service oriented architecture, Information Technology in Biomedicine, IEEE Transactions on, 2007, 11(6): 639-650

[9] Patra D, Ray S, Mukhopadhyay J, Majumdar B and Majumdar A K. Achieving e-health care in a distributed EHR system, presented at the 11th International Conference on e-Health Networking, Applications and Services. 2009: 101-107.

[10] Lopez DM and Blobel BGM. A development framework for semantically interoperable health information systems. International Journal of Medical Informatics. 2009; 78(2): 83-103.

[11] Ministerio de Salud de Colombia. Registros médicos e Historia Clínica. Bogotá; 1983

[12] Reglamento General de Archivos. Acuerdo 07 de 1994. 1994.

[13] Ministerio de Salud. Resolución Número 2546 de 1998. 1998.

[14] R El Congreso de Colombia. Ley 143819 de enero de 2011. 2011.

[15] Taylor N, Medvidović M and Dashofy EM. Software architecture: foundations, theory, and practice. Malden: John Wiley; 2009 .

[16] Gomaa H. Software Modeling and Design: UML, Use Cases, Patterns, and Software Architectures. Boston: Cambridge University Press; 2011.

[17] Apache, Introducing Axis2. [Online]. Available: http://axis.apache.org/axis2/java/core/docs/userguide.html\#intro. [Accessed: 25-may-2012].

[18] MySQL, MySQL 5.6 Reference Manual :: 1 General Information. [Online]. Available: http://dev.mysql.com/$\mathrm{doc} / \mathrm{refman} / 5.6 / \mathrm{en} /$ introduction.html. [Accessed: 25-may2012].

[19] The Apache Software Foundation, Apache Tomcat - Welcome! [Online]. Available: http://tomcat.apache.org/index.html. [Accessed: 25-may-2012].

[20] ICEsoft Technologies, ICEfaces Overview. [Online]. Available: http://wiki.icesoft.org/display/ICE/ICEfaces+Overview. [Accessed: 25-may-2012].

[21] Wikipedia contributors. XSLT. [Online]. Available: http://en.wikipedia.org/wiki/XSLT. [Accessed: 09-may2012].

[22] Kitchenham BA and Pickard LM. Evaluating software eng. methods and tools part 10: designing and running a quantitative case study. SIGSOFT Softw. Eng. Notes. 1998; 23(3): 20-22. 